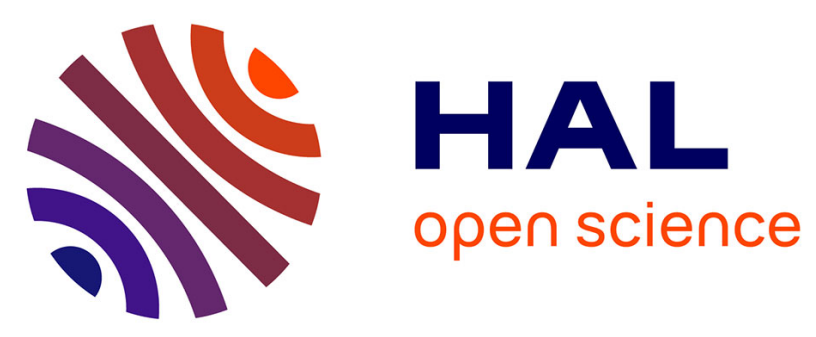

\title{
Early childcare type predicts children's emotional and behavioural trajectories into middle childhood. Data from the EDEN mother-child cohort study
}

\author{
Ramchandar Gomajee, Fabienne El-Khoury, Sylvana Côté, Judith van Der
} Waerden, Laura Pryor, Maria Melchior

\section{To cite this version:}

Ramchandar Gomajee, Fabienne El-Khoury, Sylvana Côté, Judith van Der Waerden, Laura Pryor, et al.. Early childcare type predicts children's emotional and behavioural trajectories into middle childhood. Data from the EDEN mother-child cohort study. Journal of Epidemiology and Community Health, 2018, 72 (11), pp.1033-1043. 10.1136/jech-2017-210393 . hal-02052401

\section{HAL Id: hal-02052401 \\ https://hal.sorbonne-universite.fr/hal-02052401}

Submitted on 28 Feb 2019

HAL is a multi-disciplinary open access archive for the deposit and dissemination of scientific research documents, whether they are published or not. The documents may come from teaching and research institutions in France or abroad, or from public or private research centers.
L'archive ouverte pluridisciplinaire HAL, est destinée au dépôt et à la diffusion de documents scientifiques de niveau recherche, publiés ou non, émanant des établissements d'enseignement et de recherche français ou étrangers, des laboratoires publics ou privés. 


\section{Early childcare type predicts children's emotional and behavioural}

\section{trajectories into middle childhood. Data from the EDEN mother-child}

\section{cohort study.}

Ramchandar Gomajee, $\mathrm{MSc}^{1}$; Fabienne El-Khoury, $\mathrm{PhD}^{1}$; Sylvana M. Côté, $\mathrm{PhD}^{2,3}$; Judith van der Waerden, $\mathrm{PhD}^{1}$; Laura Pryor, $\mathrm{PhD}^{1}$; Maria Melchior, $\mathrm{PhD}^{1}$.

1. Sorbonne Universités, UPMC Paris 06, INSERM UMRS 1136, Institut Pierre Louis d'épidémiologie et de Santé Publique (IPLESP), 75012, Paris, France

2. INSERM U1219, Université de Bordeaux, Bordeaux, France

3. Département de Médecine Sociale et Préventive, Université de Montréal, Canada

\section{Corresponding author:}

Ramchandar Gomajee

Email address: ramchandar.gomajee@inserm.fr

Address: INSERM U1136 / IPLESP,

Université Pierre et Marie Curie site St. Antoine,

27 rue Chaligny,

75012 Paris, France

Phone number: 0686255421

Word count: 3629

Tables: 3

Figures: 2

References: 42

Keywords: child care, cohort, behavioural symptoms, France, childhood. 


\section{ABSTRACT}

Background: The scientific literature on the impact of early childcare on children's behavioural and emotional difficulties shows contrasting results. We studied this association in France, where childcare is of high quality and children enter preschool at the age of three.

Methods: 1428 children from the EDEN mother-child cohort set up in France (Nancy and Poitiers) were followed up since pregnancy to age 8 years. Group-based trajectory modelling was used to model their trajectories of behavioural and emotional symptoms (emotional symptoms, peer relationship problems, hyperactivity-inattention, conduct problems, prosocial behaviours) ascertained by three measures (3, 5.5 and 8 years) of the Strengths and Difficulties Questionnaires. Using propensity scores and Inverse Probability Weights to account for selection and confounding factors we compared children in a childminder's care or in centre-based childcare (from birth to age 3) to those in informal childcare.

Results: Compared to children in informal childcare, those who attended centre-based childcare had a lower likelihood of having high levels of emotional $\left(\mathrm{OR}_{\mathrm{IPW} \text {-adjusted }}=0.35,95 \%\right.$ CI: 0.17-0.71), peer relationship problems $\left(\mathrm{OR}_{\mathrm{IPW}-\text { adjusted }}=0.31,95 \% \mathrm{CI}: 0.15-0.67\right)$, and low prosocial behaviours $\left(\mathrm{OR}_{\mathrm{IPW} \text {-adjusted }}=0.50,95 \% \mathrm{CI}\right.$ : 0.28-0.90). Those who were looked after by a childminder had a higher likelihood of following a high trajectory of conduct problems $\left(\mathrm{OR}_{\mathrm{IPW} \text {-adjusted }}=1.72,95 \% \mathrm{CI} 1.05-2.81\right)$. Attendance of centre-based childcare for more than one year was especially protective of high levels of emotional, peer-related difficulties and low prosocial behaviours. Girls and children from a favourable socioeconomic background reaped more benefits of childcare than boys and those from a less favourable background.

Conclusion: High quality centre-based childcare may be linked to lower levels of emotional symptoms.

\section{SUMMARY BOX}




\section{What is already known on this subject?}

The scientific literature on the relationship between childcare and behavioural and emotional difficulties shows contrasting results. More recent studies suggest that if childcare is of high quality, it is associated with lower levels of symptoms, especially in children growing up in socioeconomically disadvantaged families.

\section{What does this study add?}

We studied this topic in France, where early childcare is subsidised and universally accessible. Children's behavioural and emotional symptoms in middle childhood (3 to 8 years) were studied in relation to childcare during the first 3 years of life (centre-based, childminder, informal care). Formal childcare in the first three years of life predicts a lower likelihood of high levels of behavioural and emotional symptoms in middle childhood, especially in children who were in centre-based childcare. This was particularly true for emotional symptoms and peer-relationship problems. Those who were in centre-based childcare for at least one year benefitted the most, as did girls and children from socioeconomically disadvantaged backgrounds. Overall, high quality centre-based childcare specifically predicts lower levels of emotional difficulties, peer problems, and low prosocial behaviours into middle-childhood. 


\section{INTRODUCTION}

\subsection{Background}

Early childcare has a positive impact on children's academic readiness as well as cognitive, language and pre-academic skills.[1,2] However, its impact on behaviour is debated.[3,4]

While some studies reported that childcare prior to school entry was associated with elevated levels of behavioural difficulties, [5,6] particularly externalizing problems, [5] others found no such effects.[7] To the contrary, other researchers found no negative effect of childcare on children's behaviour,[8] or only in the very short-term.[4] Finally, some studies suggested that the protective effects of early childcare are strongest among children growing up in high-risk families.[9]

Existing discrepancies in prior studies may result from several factors: varying childcare policies, childcare quality, age(s) at which children's behaviour was ascertained, and the use of parental vs. teacher reports of children's behaviour. Studies tend to compare either centrebased childcare to informal care, i.e. childcare by non-professional caregivers such as grandparents, relatives, friends or neighbours, or formal care, i.e. childcare by a professional, to informal care. Few studies were conducted in countries with universal childcare policy. Policies concerning early childcare differ from country to country and therefore not only may the age at which children enter preschool differ but also the time spent in childcare, its quality and availability. Furthermore, with notable exceptions,[6] prior studies only focused on short-term behavioural, and sometimes emotional difficulties, and little is known about the potential long-term consequences of early life childcare.

France provides an interesting setting to examine this question: a) the number of places in formal childcare for children under three years of age is high (approximately 52\% of children 
can attend compared to $33 \%$ on average in Europe);[10] b) childcare is of high quality (UNICEF Report Card);[11] c) nearly all children enter the formal school system at 3 years of age (97\% start by age 3 and $99 \%$ by age 4$)$, [12] making it possible to isolate the role of childcare prior to age 3; d) early childcare is based on the principle of universality, i.e. all children, irrespective of their background can access.

French families tend to use two different types of formal childcare: centre-based care (approximately $27 \%$ of children) and childminders, professional caregivers with a state degree, authorised to take care of 2 to 6 children in their own home (approximately $49 \%$ of children).[10,13] Childcare is subsidised and the two types have similar cost but vary in terms of educational content, centre-based care being generally more structured and standardised than care provided by a childminder. The number of available places is under the supervision of municipalities and varies across place and time.[13] Centre-based childcare, highly regarded by families, is in high demand. However, the number of places is limited and the hours are not always flexible, leading some families to turn to childminders.[14]

Taking advantage of this unique, quasi-experimental, situation, where families choose whether or not their child attends childcare but where the type of childcare is determined by availability, we test associations between childcare type and children's trajectories of behavioural and emotional symptoms between ages 3 and 8, using data from the mother-child EDEN cohort study. Contrary to other studies where childcare was defined as care received up to the age of 5 to 7 years, we aimed to study whether childcare in the first 3 years of life is linked to behavioural and emotional levels between ages 3 and 8 . We further investigated whether time spent in childcare $(<1$ year vs. $>=1$ year $)$ plays a role. 
Finally, based on studies showing that childcare use and influence vary depending on maternal education and psychological, as well as the child's gender,[15-17] we test whether the effects on behaviour vary across these 3 characteristics.

\section{METHODS}

\subsection{Study design, setting and participants}

The EDEN mother-child cohort received approval from the ethics committee (CCPPRB) of Kremlin Bicêtre on 12 December 2002 and from CNIL (Commission Nationale de l'Informatique et des Libertés), the French data privacy institution. It included 2002 women recruited at 24 weeks of amenorrhea in two maternity wards in two cities in France: Nancy and Poitiers (2003-2006). All women $(\mathrm{N}=3758)$ who visited the prenatal clinic of the local university hospitals were invited to take part in the study. Exclusion criteria included multiple pregnancies, known diabetes, French illiteracy and mothers planning to leave the area in the following 3 years.[18] 1,907 women with a live birth agreed (95 refusals) to take part in a regular follow-up of their child. Participating children underwent 4 clinical examinations: at

birth, 1 year, 3 years and during the $6^{\text {th }}$ year (average of 5 years and 8 months). Self-reported questionnaires were administered during these examinations, as well as in-between (at 4 months, 8 months, 2 years, and 8 years of age). Compared to the general population, mothers participating in the EDEN cohort were slightly more educated, urban, and from higher income households than non-participants. Children's characteristics such as preterm births or admission to neonatal care unit were however of the same level.[18]

Childcare use depends not only on measurable factors, such as the number of childcare places available, parents' financial and employment status, but also on unmeasurable factors, such as 
personal preference. We excluded children with no information on childcare and behaviour between ages 3 and 8 years, resulting in a final analytical sample of 1,428 children.

Compared to families included in our analysis, those who did not participate were characterized by lower maternal $(12.86, \mathrm{SD}=2.67$ vs. $13.92, \mathrm{SD}=2.59$ years; $\mathrm{p}<0.0001)$ and paternal $(12.46, \mathrm{SD}=2.54$ vs. $13.28, \mathrm{SD}=2.63$ years; $\mathrm{p}<0.0001)$ educational level, higher maternal (31.6 vs. 18.4\%; $\mathrm{p}<0.0001)$ and paternal (13.6 vs. 5.9\%; $\mathrm{p}<0.0001)$ levels of unemployment, higher family financial difficulties (61.0 vs. 50.7\%; $\mathrm{p}<0.0001)$, and lower maternal social support $(64 \%$ vs. $71.5 \% ; \mathrm{p}=0.002)$; however there were no significant differences in terms of children's sex, birth weight, prematurity or parental history of childhood behavioural difficulties.

\subsection{Variables}

\subsubsection{Childcare}

Data about childcare arrangements in the first 3 years of life were reported by mothers at 4,8 , and 12 months, and at 2 and 3 years of age. Based on this information, we created three mutually exclusive groups:

1. Childminder, CM $(636,44.5 \%)$.

2. Centre-based childcare, CBC (367, 25.7\%): day-care centre, day nursery or crèche staffed with professionals.

3. Informal childcare, IC (425, 29.8\%): primarily parents exclusively $(71.3 \%)$, sometimes complemented by grand-parents or other non-professional caregivers (e.g. other relatives, friends, neighbours, occasional babysitters).

If the mother reported that the child was in centre-based care on at least one assessment, children were included in the 'centre-based childcare' group. If not, and if children were looked after by a childminder on at least one assessment, they were included in the 
'childminder' group. Children who were looked after exclusively by their parents $(n=303$, $21.2 \%)$ or by their parents along with other non-professional caregivers $(\mathrm{n}=122,8.5 \%)$ had similar characteristics and outcomes and were grouped in 'informal care'. Transitions from one type of childcare to another (mainly from informal care to either type of formal care) primarily occurred during the first year of life $(n=823)$.

Depending on the type of childcare reported at each time point, we found that children either spent a few months in childcare $(<1$ year) or several months $(>=1$ year $)$. To take into account time spent in childcare from birth to age three, in additional analyses, children were classified in 5 groups, using 1 year in childcare as cut-off $(\mathrm{CM}<1$ year; $\mathrm{CM}>=1$ year; $\mathrm{CBC}<1$ year; $\mathrm{CBC}>=1$ year; IC).

\subsubsection{Childhood behavioural and emotional development: SDQ score}

To ascertain children's emotional and behavioural patterns we used the French version of the Strengths and Difficulties Questionnaire (SDQ) reported by the children's mother at ages 3, 5.5, and 8 years.[19]

The SDQ includes 25 items which make up 5 scales of 5 items each: one positive (pro-social behaviours) and four negative (emotional symptoms, conduct problems, symptoms of hyperactivity/inattention and peer relationship problems). Each scale ranges from 0 to 10 points with higher scores representing more problematic behaviours/symptoms except in the case of prosocial behaviours.[20]

\subsubsection{Covariates}

Characteristics potentially associated with childcare type or children's psychological development and ascertained prior to age 3 years were studied as covariates. 
These include:

- Study centre (Nancy or Poitiers): there were no differences in the methods of assessment between the two centres, but Nancy, an urban environment, has a higher density of childcare centres than Poitiers, therefore the probability of being in centre based childcare differs between these two settings;

- Child characteristics: sex, birth weight (<vs. >=2500g), premature birth $(<\mathrm{vs.}>=37$ weeks of amenorrhea), birth order (firstborn vs. not firstborn), maternal age at delivery, duration of breast-feeding (in months), and age at preschool entry;

- Parents' socio-demographic characteristics measured longitudinally between pregnancy and age 3: marital status (single vs. married/cohabiting), partner support (the mother reported whether she felt her partner was supportive, at inclusion: yes vs. no), parental educational level (< vs. $>=$ higher education), parental employment status (unemployed vs. employed), family financial difficulties (to pay for medical care, food or electricity at least once during the first 3 years of life, yes vs. no), family income (< vs. $>=1500$ euros/month), parents' activities with the child (e.g. playing, reading stories, etc. between 4 months and 2 years: almost daily, often or rarely);[21,22]

- Parents' mental health: maternal depression during pregnancy, identified using the 20 item version of the Centre for Epidemiological Studies Depression scale (CESD)(Cronbach's alpha $=0.88$ ), and dichotomized using a cut-off of 23;[23] maternal postnatal depression ascertained using the Edinburgh Postnatal Depression Scale $($ EPDS $)($ Cronbach's alpha $=0.85)$ at 4, 8 and 12 months post-partum and dichotomized using a threshold of $13 ;[24]$ maternal treatment of mental health difficulties (e.g. use of tranquilizers, antidepressants, sleeping aids, counselling) during the follow-up; maternal history of psychiatric problems prior to pregnancy; parental 
history of childhood behavioural difficulties and maternal use of psychoactive substances in pregnancy and afterwards (cigarettes, cannabis, alcohol).

\subsection{Statistical methods}

All analyses were performed with SAS, version 9.4.

Children may have fluctuating levels of symptoms at the 3 times SDQ was reported, i.e. some children may have high levels at 3 years but then decrease over time while for others it may be the opposite or stable. To identify groups of children with similar patterns of SDQ symptoms over time, we used group-based trajectory modelling.[25] The best-fitting model for each SDQ subscale was defined based on a maximized BIC (Bayesian Information Criterion) and group parsimony.[25]

On average, $6.4 \%$ of data on study covariates were missing (with a maximum of $22 \%$ for maternal depression at 12 months). Missing data were handled using the multiple imputation (MI) technique with the Fully Conditional Specification (FCS) method [26](10 imputations). The imputation model included all variables included in our substantive analysis or predictive of missingness.[27]

To render the three different childcare groups as similar as possible we used propensity scores, calculated based on all observed variables associated with childcare or behavioural difficulties. [28] All variables predictive of children's behavioural difficulties and/or childcare attendance were used to calculate propensity scores using multinomial logistic regression.

Propensity scores were included in our statistical models via inverse probability weights (IPWs), which are the reciprocal of receiving the predicted exposure.[28] Extreme weights were curtailed at the 99th percentile and the stabilised mean weight was approximately 1 . 
Finally, we verified that confounding factors were more evenly distributed across the 3 childcare groups after applying the IPWs.[28,29]

To test whether childcare type predicts children's trajectories of emotional and behavioural symptoms, we carried out multinomial logistic regression analyses adjusted for IPWs.

Additionally, we also tested whether children's behaviour varied with the time spent in formal childcare (<vs. $>=1$ year). Finally, we tested for statistical interactions between childcare type and (i) child sex, (ii) maternal educational level, (iii) maternal depression and stratified on these factors.

\section{RESULTS}

\subsection{Descriptive analysis}

In our study population (Table 1), $48.0 \%$ of children were female and the average age at preschool entry was 3.45 years $(\mathrm{SD}=0.6)$. Average SDQ scores were $9.94(\mathrm{SD}=4.8)$ at 3 years, $8.75(\mathrm{SD}=5.2)$ at 5.5 years and $8.62(\mathrm{SD}=5.2)$ at 8 years. Overall, characteristics of children in centre-based care or cared for by a childminder were similar, while children in informal care systematically had systematically less favourable profiles. 
Table 1: Characteristics of the study population $(\mathrm{N}=1428)$, means (SD) or $\mathrm{N}(\%)$; $\mathrm{p}$ for differences across childcare types.

\begin{tabular}{|c|c|c|c|c|}
\hline $\begin{array}{c}\text { Variables } \\
\% \\
\text { (N) }\end{array}$ & $\begin{array}{l}\text { Child-minder } \\
\begin{array}{c}44.5 \% \\
(\mathrm{~N}=636)\end{array}\end{array}$ & $\begin{array}{c}\text { Centre-based } \\
\begin{array}{c}25.7 \% \\
(\mathrm{~N}=367)\end{array}\end{array}$ & $\begin{array}{c}\text { Informal } \\
\text { childcare } \\
29.8 \% \\
(\mathrm{~N}=425) \\
\end{array}$ & $p$ \\
\hline \multicolumn{5}{|l|}{ Child characteristics } \\
\hline Centre of birth (Nancy) & 43.1 & 55.0 & 49.2 & 0.0011 \\
\hline Gender (Female) & 47.3 & 51.8 & 45.7 & 0.2071 \\
\hline Mother's age (years) & $29.8(4.3)$ & $29.8(4.7)$ & $30.2(5.4)$ & 0.2797 \\
\hline Birth weight $<2500 \mathrm{~g}$ & 4.1 & 6.0 & 4.7 & 0.3927 \\
\hline Premature child & 5.5 & 6.0 & 5.2 & 0.8807 \\
\hline Breastfeeding duration (months) & $2.84(3.37)$ & $3.94(4.22)$ & $3.98(5.39)$ & $<0.0001$ \\
\hline First-born & 47.5 & 48.2 & 16.5 & $<0.0001$ \\
\hline Age of entry in school (years) & $3.41(0.58)$ & $3.44(0.60)$ & $3.51(0.66)$ & 0.0234 \\
\hline \multicolumn{5}{|l|}{ Parental employment status } \\
\hline Non-working mother at 24 weeks of pregnancy & 7,9 & 11,4 & 40,7 & $<0.0001$ \\
\hline Non-working mother at 1 year & 6,1 & 11,7 & 42,3 & $<0.0001$ \\
\hline Non-working mother at 2 years & 4,4 & 7,6 & 42,6 & $<0.0001$ \\
\hline Non-working at 24 weeks of pregnancy & 4,6 & 9,0 & 8,9 & 0.0052 \\
\hline \multicolumn{5}{|l|}{ Parental educational level } \\
\hline Low educated mother ${ }^{a}$ & 43,8 & 30,2 & 61,6 & $<0.0001$ \\
\hline Low educated father ${ }^{a}$ & 49,4 & 40,9 & 64,2 & $<0.0001$ \\
\hline \multicolumn{5}{|l|}{ Maternal psychological status } \\
\hline Depression (CESD)during pregnancy ${ }^{\mathbf{b}}$ & 6.3 & 5.5 & 6.0 & $<0.0001$ \\
\hline Depression (EPDS) at 12 months $^{c}$ & 4.7 & 6.0 & 30.8 & $<0.0001$ \\
\hline Psychological help during pregnancy & 12.7 & 14.4 & 12.0 & 0.5799 \\
\hline Psychological help at 1 year & 7.9 & 10.6 & 6.8 & 0.1352 \\
\hline Psychological help at 2 years & 10.9 & 15.8 & 8.7 & 0.0060 \\
\hline History of mental health problems before pregnancy & 12.6 & 14.4 & 16.0 & 0.2836 \\
\hline Lack of social support & 25.2 & 29.4 & 34.2 & 0.0256 \\
\hline \multicolumn{5}{|l|}{ Single parent status } \\
\hline Single mother at 24 weeks of pregnancy & 1.6 & 3.5 & 3.5 & 0.0279 \\
\hline Single mother at 1 year & 1.6 & 5.7 & 3.5 & 0.0015 \\
\hline Single mother at 2 years & 3.0 & 5.2 & 5.4 & 0.0977 \\
\hline \multicolumn{5}{|l|}{ Maternal substance use } \\
\hline Cannabis use prior pregnancy & 4.9 & 5.7 & 7.3 & 0.2551 \\
\hline Tobacco use prior pregnancy & 31.6 & 29.4 & 36.0 & 0.1236 \\
\hline Tobacco use during pregnancy & 22.5 & 18.3 & 27.5 & 0.0078 \\
\hline
\end{tabular}




\begin{tabular}{|l|c|c|c|c|}
\hline Alcohol use during pregnancy & 29.9 & 26.4 & 31.5 & 0.2797 \\
\hline Financial difficulties & & & & \\
Household income < 1500 E during pregnancy & 1.7 & 4.1 & 9.2 & $<0.0001$ \\
\hline At least 1 financial difficulty (pregnancy) & 47.3 & 49.6 & 56.7 & 0.0100 \\
\hline Low household income (1st year) & 4.9 & 9.3 & 31.8 & $<0.0001$ \\
\hline At least 1 financial difficulty (1st year) & 23.9 & 25.9 & 43.3 & $<0.0001$ \\
\hline Low household income (2nd year) & 3.9 & 8.2 & 32.0 & $<0.0001$ \\
\hline At least 1 financial difficulty (2nd year) & 50.0 & 55.0 & 63.1 & 0.0002 \\
\hline Parental childhood difficulties & & & & \\
\hline Childhood adversity (mother) & 24.2 & 28.6 & 31.3 & 0.0343 \\
Childhood speech delay (mother) & 11.5 & 14.7 & 22.4 & $<0.0001$ \\
Childhood behaviour problems (mother) & 6.1 & 5.5 & 7.5 & 0.4633 \\
\hline Childhood behaviour problems (father) & 9.4 & 10.4 & 16.0 & 0.0032 \\
\hline Childhood speech delay (father) & 26.3 & 24.8 & 41.7 & $<0.0001$ \\
\hline Child activities with the parents & & & & \\
\hline Daily activities < 1 year (mother) & 82.1 & 80.7 & 55.1 & $<0.0001$ \\
\hline Daily activities at 1 year (mother) & 92.8 & 91.3 & 94.6 & 0.1899 \\
\hline Daily activities at 1 year (father) & 78.8 & 77.9 & 70.8 & 0.0078 \\
\hline Daily activities at 2 years (mother) & 51.3 & 66.8 & 77.2 & $<0.0001$ \\
\hline Daily activities at 2 years (father) & 72.2 & 64.0 & 80.0 & $<0.0001$ \\
\hline
\end{tabular}

${ }^{a}$ Low level of education: No tertiary education (high school diploma or lower)

${ }^{\mathrm{b}}$ Centre of Epidemiological Studies Depression scale

${ }^{\mathrm{c}}$ Edinburg Postnatal Depression Scale 


\subsection{Childcare type predicts children's emotional and behavioural trajectories}

Figure 1 shows children's trajectories of emotional and behavioural symptoms between ages 3 and 8 years. For each SDQ subscale, the best model predicts three distinct trajectories representing three levels of symptoms (low, intermediate and high level symptoms). Children belonging to the 'low level symptoms' group served as the reference category, except for prosocial behaviours where the 'high level symptoms' group was the reference.

The majority of children had low or intermediate level symptom trajectories (except for prosocial behaviours). Overall $15.5 \%$ had persistently high levels of conduct problems, $15.1 \%$ high symptoms of hyperactivity/inattention, $16.0 \%$ of emotional symptoms, $6.8 \%$ of peer relationship problems and $13.1 \%$ had persistently low levels of prosocial behaviours.

In bivariate analyses (Table 2), compared to children in informal childcare, those attending centre-based childcare were less likely to have high levels of emotional symptoms, peer relationship problems, hyperactivity/inattention and conduct problems, while those cared for by a childminder were less likely to have high levels of peer relationship problems. After controlling for child and family characteristics via IPW (Table 2), compared to children in informal childcare, those who attended centre-based childcare were less likely to have high levels of emotional $\left(\mathrm{OR}_{\mathrm{IPW} \text {-adjusted }}=0.35,95 \% \mathrm{CI}\right.$ : 0.17-0.71) and peer relationship problems $\left(\mathrm{OR}_{\mathrm{IPW} \text {-adjusted }}=0.31,95 \%\right.$ CI $\left.0.15-0.67\right)$. Children looked after by a childminder were somewhat more likely to follow a high trajectory of conduct problems $\left(\mathrm{OR}_{\mathrm{IPW} \text {-adjusted }}=1.72\right.$ $[1.05-2.81])$. 
Table 2: Early childcare (childminder, centre-based childcare or informal care) from 0 to 3 years and children's trajectories of behavioural and emotional difficulties (low, intermediate or high) from ages 3 to 8 years; EDEN cohort study ( $n=1428)$, bivariate multinomial regression and Inverse Probability Weight (IPW)-adjusted analyses (95\% CI); reference group $=$ informal care.

\begin{tabular}{|c|c|c|c|c|c|c|c|c|c|c|c|c|c|}
\hline & & \multicolumn{6}{|c|}{ Bivariate analysis } & \multicolumn{6}{|c|}{ IPW-adjusted analysis } \\
\hline \multirow{2}{*}{\multicolumn{2}{|c|}{ SDQ subscales }} & \multicolumn{3}{|c|}{ CM vs. IC } & \multicolumn{3}{|c|}{ CBC vs. IC } & \multicolumn{3}{|c|}{ CM vs. IC } & \multicolumn{3}{|c|}{ CBC vs. IC } \\
\hline & & OR & $95 \%$ IC & $p$ & OR & $95 \%$ IC & $p$ & $O R$ & $95 \%$ IC & $p$ & $O R$ & $95 \%$ IC & $p$ \\
\hline \multirow{3}{*}{$\begin{array}{l}\text { Emotional } \\
\text { Symptoms }\end{array}$} & $L$ & \multicolumn{3}{|c|}{ Reference } & \multicolumn{3}{|c|}{ Reference } & \multicolumn{3}{|c|}{ Reference } & \multicolumn{3}{|c|}{ Reference } \\
\hline & $I$ & 0.80 & $0.54-1.19$ & 0.2715 & 0.58 & $0.39-0.88$ & 0.0102 & 0.41 & $0.22-0.79$ & 0.0080 & 0.33 & $0.18-0.60$ & 0.0004 \\
\hline & $H$ & 1.04 & $0.65-1.67$ & 0.8638 & 0.54 & $0.32-0.92$ & 0.0229 & 0.58 & $0.31-1.08$ & 0.0882 & 0.35 & $0.17-0.71$ & 0.0037 \\
\hline \multirow{3}{*}{$\begin{array}{c}\text { Peer } \\
\text { Relationship } \\
\text { Problems }\end{array}$} & $L$ & \multicolumn{3}{|c|}{ Reference } & \multicolumn{3}{|c|}{ Reference } & \multicolumn{3}{|c|}{ Reference } & \multicolumn{3}{|c|}{ Reference } \\
\hline & $I$ & 0.67 & $0.47-0.95$ & 0.0240 & 0.49 & $0.34-0.72$ & 0.0002 & 0.98 & $0.61-1.58$ & 0.9453 & 0.78 & $0.50-1.21$ & 0.2680 \\
\hline & $H$ & 0.47 & $0.27-0.82$ & 0.0079 & 0.33 & $0.17-0.62$ & 0.0007 & 0.52 & $0.24-1.12$ & 0.0930 & 0.31 & $0.15-0.67$ & 0.0032 \\
\hline \multirow{3}{*}{$\begin{array}{l}\text { Hyperactivity } \\
\text { / Inattention }\end{array}$} & $L$ & \multicolumn{3}{|c|}{ Reference } & \multicolumn{3}{|c|}{ Reference } & \multicolumn{3}{|c|}{ Reference } & \multicolumn{3}{|c|}{ Reference } \\
\hline & $I$ & 0.89 & $0.67-1.19$ & 0.4404 & 0.71 & $0.52-0.98$ & 0.0371 & 1.34 & $0.86-2.11$ & 0.1927 & 1.18 & $0.75-1.87$ & 0.4651 \\
\hline & $H$ & 0.97 & $0.66-1.42$ & 0.8556 & 0.50 & $0.32-0.81$ & 0.0041 & 1.53 & $0.98-2.38$ & 0.0593 & 1.02 & $0.54-1.95$ & 0.9460 \\
\hline \multirow{3}{*}{$\begin{array}{l}\text { Conduct } \\
\text { Problems }\end{array}$} & $L$ & \multicolumn{3}{|c|}{ Reference } & \multicolumn{3}{|c|}{ Reference } & \multicolumn{3}{|c|}{ Reference } & \multicolumn{3}{|c|}{ Reference } \\
\hline & $I$ & 0.91 & $0.67-1.25$ & 0.5699 & 0.72 & $0.51-1.01$ & 0.0564 & 1.07 & $0.75-1.52$ & 0.7087 & 1.00 & $0.71-1.39$ & 0.9789 \\
\hline & $H$ & 0.72 & $0.48-1.08$ & 0.1121 & 0.54 & $0.34-0.85$ & 0.0085 & 1.72 & $1.05-2.81$ & 0.0306 & 1.36 & $0.67-2.78$ & 0.3777 \\
\hline \multirow{3}{*}{$\begin{array}{c}\text { Prosocial } \\
\text { Behaviours }\end{array}$} & $L$ & 1.20 & $0.79-1.84$ & 0.3924 & 0.67 & $0.41-1.10$ & 0.1137 & 1.16 & $0.71-1.89$ & 0.5628 & 0.81 & $0.50-1.30$ & 0.3797 \\
\hline & $I$ & 1.16 & $0.85-1.57$ & 0.3511 & 0.83 & $0.59-1.16$ & 0.2684 & 0.88 & $0.58-1.32$ & 0.5222 & 0.66 & $0.47-0.94$ & 0.0207 \\
\hline & $H$ & \multicolumn{3}{|c|}{ Reference } & \multicolumn{3}{|c|}{ Reference } & \multicolumn{3}{|c|}{ Reference } & \multicolumn{3}{|c|}{ Reference } \\
\hline
\end{tabular}

$\mathbf{C M}=$ Childminder (45.5\%); $\mathbf{C B C}=$ Centre-based childcare (25.7\%); IC = Informal care (29.8\%).

$L=$ low-level symptoms; $I=$ intermediate-level symptoms, $H=$ high-level symptoms. 
Among children who were in formal childcare, those who attended centre-based childcare had the lowest levels of emotional symptoms and peer relationship problems irrespective of duration (Table 3) (respectively: $<1$ year: high emotional symptoms: $\mathrm{OR}_{\mathrm{IPW} \text {-adjusted }}=0.23$, 95\% CI: 0.11-0.50 and high peer relationship problems: $\mathrm{OR}_{\mathrm{IPW} \text {-adjusted }}=0.14,95 \%$ CI: 0.05 $0.39 ;>=1$ year: high emotional symptoms: $\mathrm{OR}_{\mathrm{IPW}-\text { adjusted }}=0.27,95 \% \mathrm{CI}: 0.12-0.58$ and high peer relationship problems: $\left.\mathrm{OR}_{\mathrm{IPW} \text {-adjusted }}=0.43,95 \% \mathrm{CI}: 0.20-0.91\right)$. This was also the case of children who spent one year or more with a childminder (high emotional symptoms: $\left.\mathrm{OR}_{\mathrm{IPW} \text {-adjusted }}=0.38,95 \% \mathrm{CI}: 0.18-0.80\right)$ and high peer relationship problems: $\mathrm{OR}_{\mathrm{IPW} \text {-adjusted }}=$ 0.32, 95\% CI: 0.13-0.76). However, only children who spent at least one year in centre-based childcare had a decreased likelihood of having high levels of hyperactivity/inattention $\left(\mathrm{OR}_{\mathrm{IPW} \text {-adjusted }}=0.42,95 \% \mathrm{CI}: 0.22-0.82\right)$ and low levels of prosocial behaviours $\left(\mathrm{OR}_{\mathrm{IPW} \text {-adjusted }}\right.$ $=0.50,95 \%$ CI $0.28-0.90)$.

Table 3: Time in early childcare (childminder, centre-based childcare or informal care) from 0 to 3 years and children's behavioural and emotional difficulties from ages 3 to 8 years; EDEN cohort study $(\mathrm{n}=1428)$, bivariate multinomial regression and Inverse Probability Weight (IPW)-adjusted analyses (95\% CI); reference group = informal care .

\begin{tabular}{|c|c|c|c|c|c|c|c|c|c|c|c|c|c|}
\hline \multirow{2}{*}{\multicolumn{2}{|c|}{$\begin{array}{l}\text { SDQ sub-scale \& } \\
\text { trajectories }\end{array}$}} & \multicolumn{3}{|c|}{$\mathrm{CM}<1$ year vs. IC } & \multicolumn{3}{|c|}{ CM >= 1 year vs. IC } & \multicolumn{3}{|c|}{$\mathrm{CBC}<1$ year vs. $\mathrm{IC}$} & \multicolumn{3}{|c|}{ CBC > = 1 year vs. IC } \\
\hline & & OR & $95 \%$ IC & $p$ & OR & $95 \%$ IC & $p$ & OR & $95 \%$ IC & $p$ & OR & $95 \%$ IC & $p$ \\
\hline \multicolumn{14}{|c|}{ BIVARIATE ANALYSES } \\
\hline \multirow{3}{*}{$\begin{array}{l}\text { Emotional } \\
\text { Symptoms }\end{array}$} & $L$ & \multicolumn{3}{|c|}{ Reference } & \multicolumn{3}{|c|}{ Reference } & \multicolumn{3}{|c|}{ Reference } & \multicolumn{3}{|c|}{ Reference } \\
\hline & $I$ & 1.07 & $0.59-1.94$ & 0.8142 & 0.73 & $0.48-1.10$ & 0.1336 & 0.65 & $0.39-1.10$ & 0.1086 & $\mathbf{0 . 5 3}$ & $0.33-0.85$ & 0.0090 \\
\hline & $H$ & 1.35 & $0.67-2.72$ & 0.3971 & 0.96 & $0.58-1.58$ & 0.8676 & 0.51 & $0.25-1.02$ & 0.0558 & 0.56 & $0.30-1.04$ & 0.0680 \\
\hline \multirow{3}{*}{$\begin{array}{l}\text { Peer } \\
\text { Relationship } \\
\text { Problems }\end{array}$} & $L$ & \multicolumn{3}{|c|}{ Reference } & \multicolumn{3}{|c|}{ Reference } & \multicolumn{3}{|c|}{ Reference } & \multicolumn{3}{|c|}{ Reference } \\
\hline & $I$ & 1.13 & $0.65-1.95$ & 0.6712 & 0.57 & $0.39-0.82$ & 0.0026 & 0.61 & $0.38-0.98$ & 0.0410 & 0.42 & $0.27-0.64$ & $<0.0001$ \\
\hline & $H$ & 0.84 & $0.37-1.92$ & 0.6733 & 0.40 & $0.22-0.72$ & 0.0024 & 0.34 & $0.14-0.81$ & 0.0154 & 0.32 & $0.15-0.68$ & 0.0032 \\
\hline \multirow{3}{*}{$\begin{array}{l}\text { Hyperactivity } \\
\text { / Inattention }\end{array}$} & $L$ & \multicolumn{3}{|c|}{ Reference } & \multicolumn{3}{|c|}{ Reference } & \multicolumn{3}{|c|}{ Reference } & \multicolumn{3}{|c|}{ Reference } \\
\hline & $I$ & 1.23 & $0.80-1.90$ & 0.3440 & 0.80 & $0.58-1.08$ & 0.1463 & 0.83 & $0.55-1.26$ & 0.3857 & 0.63 & $0.44-0.92$ & 0.0160 \\
\hline & $H$ & 1.10 & $0.62-1.97$ & 0.7426 & 0.93 & $0.61-1.40$ & 0.7126 & 0.73 & $0.41-1.30$ & 0.2836 & 0.36 & $0.19-0.65$ & 0.0009 \\
\hline
\end{tabular}




\begin{tabular}{|c|c|c|c|c|c|c|c|c|c|c|c|c|c|}
\hline \multirow{3}{*}{$\begin{array}{l}\text { Conduct } \\
\text { Problems }\end{array}$} & \multirow{2}{*}{\begin{tabular}{l|}
$L$ \\
$I$
\end{tabular}} & \multicolumn{3}{|c|}{ Reference } & \multicolumn{3}{|c|}{ Reference } & \multicolumn{3}{|c|}{ Reference } & \multicolumn{3}{|c|}{ Reference } \\
\hline & & 1.39 & $0.86-2.26$ & 0.1796 & 0.80 & $0.58-1.11$ & 0.1837 & 0.78 & $0.50-1.20$ & 0.2563 & 0.68 & $0.46-1.01$ & 0.0541 \\
\hline & $H$ & 1.25 & $0.69-2.28$ & 0.4646 & 0.60 & $0.39-0.92$ & 0.0200 & 0.74 & $0.42-1.32$ & 0.3100 & 0.40 & $0.22-0.71$ & 0.0019 \\
\hline \multirow{3}{*}{$\begin{array}{l}\text { Prosocial } \\
\text { Behaviours }\end{array}$} & $L$ & 1.02 & $0.55-1.90$ & 0.9540 & 1.28 & $0.81-2.01$ & 0.2900 & 0.79 & $0.42-1.50$ & 0.4725 & 0.59 & $0.32-1.07$ & 0.0827 \\
\hline & $I$ & 1.12 & $0.72-1.74$ & 0.6108 & 1.17 & $0.84-1.63$ & 0.3942 & 0.94 & $0.61-1.45$ & 0.7770 & 0.75 & $0.51-1.11$ & 0.1512 \\
\hline & $H$ & \multicolumn{3}{|c|}{ Reference } & \multicolumn{3}{|c|}{ Reference } & \multicolumn{3}{|c|}{ Reference } & \multicolumn{3}{|c|}{ Reference } \\
\hline \multicolumn{14}{|c|}{ INVERSE PROBABILITY WEIGHT (IPW) - ADJUSTED ANALYSES } \\
\hline \multirow{3}{*}{$\begin{array}{l}\text { Emotional } \\
\text { Symptoms }\end{array}$} & $L$ & \multicolumn{3}{|c|}{ Reference } & \multicolumn{3}{|c|}{ Reference } & \multicolumn{3}{|c|}{ Reference } & \multicolumn{3}{|c|}{ Reference } \\
\hline & $I$ & 0.57 & $0.27-1.18$ & 0.1302 & 0.32 & $0.16-0.63$ & 0.0009 & 0.23 & $0.12-0.46$ & $<0.0001$ & 0.36 & $0.19-0.69$ & 0.0022 \\
\hline & $H$ & 0.92 & $0.41-2.06$ & 0.8413 & 0.38 & $0.18-0.80$ & 0.0103 & 0.23 & $0.11-0.50$ & 0.0002 & 0.27 & $0.12-0.58$ & 0.0009 \\
\hline \multirow{3}{*}{$\begin{array}{l}\text { Peer } \\
\text { Relationship } \\
\text { Problems }\end{array}$} & $L$ & \multicolumn{3}{|c|}{ Reference } & \multicolumn{3}{|c|}{ Reference } & \multicolumn{3}{|c|}{ Reference } & \multicolumn{3}{|c|}{ Reference } \\
\hline & $I$ & 1.07 & $0.64-1.81$ & 0.7927 & 0.85 & $0.51-1.40$ & 0.5179 & 0.74 & $0.45-1.22$ & 0.2340 & 0.62 & 0.38-1.02 & 0.0622 \\
\hline & $H$ & 0.62 & $0.28-1.35$ & 0.2238 & 0.32 & $0.13-0.76$ & 0.0106 & 0.14 & $0.05-0.39$ & 0.0002 & 0.43 & $0.20-0.91$ & 0.0279 \\
\hline \multirow{3}{*}{$\begin{array}{l}\text { Hyperactivity } \\
\text { / Inattention }\end{array}$} & $L$ & \multicolumn{3}{|c|}{ Reference } & \multicolumn{3}{|c|}{ Reference } & \multicolumn{3}{|c|}{ Reference } & \multicolumn{3}{|c|}{ Reference } \\
\hline & $I$ & 1.65 & $0.98-2.76$ & 0.0569 & 1.24 & $0.75-2.05$ & 0.3850 & 1.30 & $0.79-2.14$ & 0.3008 & 0.99 & $0.66-1.48$ & 0.9626 \\
\hline & $H$ & 3 & $0.78-2.61$ & 0.2413 & 1.41 & $0.83-2.38$ & 0.2019 & 1.09 & $0.62-1.94$ & 0.7625 & 0.42 & $0.22-0.82$ & 0.0105 \\
\hline \multirow{3}{*}{$\begin{array}{l}\text { Conduct } \\
\text { Problems }\end{array}$} & $L$ & \multicolumn{3}{|c|}{ Reference } & & Reference & & & Reference & & & Reference & \\
\hline & $I$ & 1.77 & $1.06-2.94$ & 0.0280 & 0.81 & $0.52-1.27$ & 0.3618 & 0.94 & $0.62-1.42$ & 0.7578 & 0.84 & $0.47-1.51$ & 0.5531 \\
\hline & $H$ & 3.52 & $1.84-6.74$ & 0.0002 & 1.31 & $0.73-2.36$ & 0.3643 & 1.54 & $0.78-3.03$ & 0.2075 & 0.78 & $0.38-1.58$ & 0.4842 \\
\hline \multirow{3}{*}{$\begin{array}{l}\text { Prosocial } \\
\text { Behaviours }\end{array}$} & $L$ & 0.78 & $0.42-1.44$ & 0.4245 & 1.56 & $0.86-2.84$ & 0.1400 & 0.94 & $0.52-1.71$ & 0.8414 & 0.50 & $0.28-0.90$ & 0.0216 \\
\hline & $I$ & 39 & $0.56-1.41$ & 0.6156 & 0.93 & $0.59-1.48$ & 0.7652 & 0.71 & $0.46-1.10$ & 0.1227 & 0.40 & $0.25-0.66$ & 0.0003 \\
\hline & $H$ & \multicolumn{3}{|c|}{ Reference } & & Reference & & & Referenc & & & Referenc & \\
\hline
\end{tabular}

$\mathbf{C M}<\mathbf{1}$ year: Childminder for less than 1 year (12.2\%); $\mathbf{C M}>=\mathbf{1}$ year: Childminder for at least 1 year (32.3\%); $\mathbf{C B C}<\mathbf{1}$ year: Centre-based childcare for less than 1 year $(11.7 \%)$; $\mathbf{C B C}>\mathbf{1} \mathbf{1}$ year: Centre-based childcare for at least 1 year (14.0\%); IC: Informal care (29.8\%).

$L=$ low-level symptoms; $I=$ intermediate-level symptoms, $H=$ high-level symptoms.

\subsection{Subgroup analyses}

Further analyses (Figure 2) showed that girls, children whose mother had higher education and those whose mother was not depressed benefited particularly from formal childcare. Compared to girls who were in informal childcare, those who were in centre-based childcare 
or with a childminder had a lower likelihood of a high trajectory of emotional symptoms and peer relationship problems. Compared to boys in informal care, those who were in centrebased childcare seemed to have fewer emotional symptoms but those in a childminder's care were more likely to have conduct problems. Only children whose mother had higher education were less likely to have high levels of emotional symptoms and peer relationship problems in case of centre-based or childminder's care, as compared to informal care. Children whose mother experienced depression were less likely to have high levels of peer relationship or emotional problems if they attended centre-based childcare.

\section{DISCUSSION}

\section{Main results}

Our study, conducted among a community sample of children in France, shows three types of trajectories in children's behavioural and emotional symptoms between ages 3 and 8: persistently high, intermediate and low levels of symptoms. Formal childcare prior to age 3 years predicts low levels of emotional symptoms, peer relationship problems and high levels of prosocial behaviours into middle-childhood, particularly if children attend for at least one year. Childcare also appears to confer benefits in terms of prosocial behaviours. The effects of centre-based, collective, childcare are stronger than those conferred by care provided by a childminder. Our data suggest that opportunities for young children's socialization and stimulation, such as those offered by quality centre-based childcare, can serve to prevent children's emotional difficulties and help develop their psychosocial skills over the long term.

\section{Limitations and strengths}


Prior to interpreting our data, we need to acknowledge several limitations. First, the EDEN study was conducted in two distinct cities in France and is not nationally representative. The study population is overall more educated has higher household income than families in the general population of France. Nevertheless, our sample is socioeconomically heterogeneous and the distribution of childcare types is comparable to the general population.[10] Second, children's psychological and behavioural symptoms were reported by the mother and may have been influenced by maternal reporting style. Though research has shown that mothers tend to report somewhat higher levels of children's problems than teachers, [30] both types of informants are generally consistent and valid, particularly in early and middle childhood.[31] Third, the time per day spent in childcare and childcare quality are not directly measured in our study. However, centre-based childcare is generally of high quality in France [32]. Time spent in formal childcare is 37-38 hours per week.[10]

Our study's main strengths are: a) a 'quasi-experimental' setting where children with similar characteristics attend either centre-based childcare or a childminder, depending on availability rather than family choice;[13] b) repeated measures of children's and families' characteristics; c) longitudinal assessments of children's psychological and behavioural characteristics from early to middle childhood;[33] d) use of propensity scores and inverse probability weights to render exposure groups strictly comparable [34] to account for selection and confounding factors. This does not entirely rule out the possibility of unmeasured confounders (e.g. contextual factors such as the local availability of childcare), but it is unlikely that these would explain the associations observed.

\section{Findings' interpretation}


Ours is one of few studies to examine the association between childcare type and children's psychological development over several years in a country with a policy of universal access to early childcare, directly comparing two different childcare types.

Our data contrast with studies reporting that non-maternal childcare is associated with children's elevated levels of behavioural problems.[8,35] However, one was based in the USA, where childcare is unsubsidized - that is very expensive - or aimed at children from disadvantaged families and of relatively low quality (the USA ranks in the bottom half of industrial countries in this area),[11] and both measured children's behaviour at one time point which can introduce bias.

However, our results are consistent with research from Norway, where childcare access and quality are similar to France.[36] Nevertheless, in contrast to Norway, in France the number of places available in centre-based childcare centres does not entirely cover demand and approximately one third of children 0-3 years are cared for by a childminder. Childminders are trained and qualified, however their activity is less regulated than that of centre-based childcare centres and the quality of care they offer is heterogeneous.[37] This might explain why in our study childminder care appears less beneficial than centre-based care. Enhancing childcare quality might have a positive impact on social and emotional development from early childhood onwards.[38]

Low levels of emotional symptoms, peer relationship problems and hyperactivity-inattention observed among children attending centre-based childcare may reflect the benefits of cognitive stimulation through play, praise and reading,[39] opportunities for socialization and acquisition of rules which can contribute to children's self-esteem,[38] as well as quality caregiver-child interactions (i.e. positive emotionality, sensitivity and responsiveness, the avoidance of harsh physical punishment).[40] The benefits of centre-based childcare in terms 
of children's development may result from the combination of these different positive elements.

\section{Interaction between maternal and child characteristics and childcare benefits.}

In our study, girls appear to reap more benefits from formal childcare than boys, particularly in terms of peer relations, emotional symptoms and prosocial behaviours. This is in line with studies showing that centre-based childcare mainly reduces internalizing problems which are most frequent in girls.[41] However, in contrast to other studies, we found no extra benefit associated with childcare for children from high-risk families.[15] To the contrary, low-risk children appeared to have especially low levels of psychological difficulties when attending childcare. It may be that the universal curriculum that is proposed to all children does not suffice to compensate the difficulties resulting from family socioeconomic or mental health difficulties.

\section{Conclusion}

Access to high quality childcare in the first years of life may improve children's emotional and cognitive development, prevent later emotional difficulties and promote prosocial behaviours. [42] Future research taking into account the daily time spent in childcare in nationally representative samples of children is needed to confirm these benefits on psychological development and whether they translate to a reduced likelihood of psychiatric disorders later in life. 
- Competing interest: None declared.

- Acknowledgement: The EDEN Mother-Child Cohort Study Group includes I. AnnesiMaesano, J. Y. Bernard, J. Botton, M.A. Charles, P. Dargent-Molina, B. de LauzonGuillain, P. Ducimetière, M. de Agostini, B. Foliguet, A. Forhan, X. Fritel, A. Germa, V. Goua, R. Hankard, B. Heude, M. Kaminski, B. Larroque†, N. Lelong, J. Lepeule, G. Magnin, L. Marchand, C. Nabet, F. Pierre, R. Slama, M.J. Saurel-Cubizolles, M. Schweitzer, O. Thiebaugeorges.

- MM and JVDW were responsible for data acquisition. MM, JVDW, FEK, LP, SMC and RG contributed to the design of the study. RG carried out the analyses and drafted the first manuscript. All authors contributed to critically interpret the results and revise the draft before the approval of the final version of the manuscript. MM coordinated the progress of the study.

- The Corresponding Author has the right to grant on behalf of all authors and does grant on behalf of all authors, an exclusive licence (or non-exclusive for government employees) on a worldwide basis to the BMJ Publishing Group Ltd and its Licensees to permit this article (if accepted) to be published in JECH editions and any other BMJPGL products to exploit all subsidiary rights, as set out in our licence. (http://group.bmj.com/products/journals/instructions-for-authors/licence-forms/). 


\section{$\underline{\text { References }}$}

1 Laurin JC, Geoffroy M-C, Boivin M, et al. Child Care Services, Socioeconomic Inequalities, and Academic Performance. Pediatrics 2015;136:1112-24. doi:10.1542/peds.2015-0419

2 Côté SM, Mongeau C, Japel C, et al. Child Care Quality and Cognitive Development: Trajectories Leading to Better Preacademic Skills.

2012.http://onlinelibrary.wiley.com/doi/10.1111/cdev.12007/abstract (accessed 25 Apr 2017).

3 Dearing E, Zachrisson HD. Concern Over Internal, External, and Incidence Validity in Studies of Child-Care Quantity and Externalizing Behavior Problems. Child Dev Perspect Published Online First: 1 January 2017. doi:10.1111/cdep.12224

4 Pingault J-B, Tremblay RE, Vitaro F, et al. Early Nonparental Care and Social Behavior in Elementary School: Support for a Social Group Adaptation Hypothesis. Child Dev 2015;86:146988. doi:10.1111/cdev.12399

5 Yamauchi C, Leigh A. Which children benefit from non-parental care? Econ Educ Rev 2011;30:1468-90. doi:10.1016/j.econedurev.2011.07.012

6 Baker M, Gruber J, Milligan K. Non-Cognitive Deficits and Young Adult Outcomes: The Long-Run Impacts of a Universal Child Care Program. National Bureau of Economic Research 2015. doi:10.3386/w21571

7 Gialamas A, Sawyer ACP, Mittinty MN, et al. Quality of childcare influences children's attentiveness and emotional regulation at school entry. J Pediatr 2014;165:813-819.e3. doi:10.1016/j.jpeds.2014.06.011

8 Datta Gupta N, Simonsen M. Non-cognitive child outcomes and universal high quality child care. J Public Econ 2010;94:30-43. doi:10.1016/j.jpubeco.2009.10.001

9 Geoffroy M-C, Côté SM, Giguère C-É, et al. Closing the gap in academic readiness and achievement: the role of early childcare. J Child Psychol Psychiatry 2010;51:1359-67. doi:10.1111/j.1469-7610.2010.02316.x

10 CNAF, Dossier de Presse. Les résultats 2012 de l'observatoire national de la petite enfance. 2013.https://www.caf.fr/sites/default/files/cnaf/Documents/DCom/Presse/Communiqu\%C3\%A9 s\%202013/14nov2013_DP_Obsevatoire.pdf (accessed 13 Jul 2017).

11 UNICEF Innocenti Research Centre. The child care transition, Innocenti Report Card 8. 2008.https://www.unicef-irc.org/publications/pdf/rc8_eng.pdf (accessed 13 Jul 2017).

12 Blanpain N. Scolarisation et modes de garde des enfants âgés de 2 à 6 ans. DREES, Études et Résultats, $\mathrm{n}^{\circ} 497$.

2006.http://www.lalettredeleducation.fr/IMG/pdf/tudes_et_resultats_no497_juin_2006.pdf (accessed 7 Feb 2017).

13 Acs $M$. Les spécificités régionales des modes de garde déclarés des enfants de moins de 3 ans. DREES études et résultats $N^{\circ}$ 839. 2013.http://drees.social-sante.gouv.fr/IMG/pdf/er839.pdf (accessed 8 Feb 2017). 
14 Fagnani J. Equal access to quality care: Lessons from France on providing high quality and affordable childcare and early education.

2012.http://sticerd.Ise.ac.uk/dps/case/events/France.pdf (accessed 12 Jul 2017).

15 Herba CM, Tremblay RE, Boivin M, et al. Maternal depressive symptoms and children's emotional problems: Can early child care help children of depressed mothers? JAMA Psychiatry 2013;70:830-8. doi:10.1001/jamapsychiatry.2013.1361

16 Petitclerc A, Côté S, Doyle O, et al. Who uses early childhood education and care services? Comparing socioeconomic selection across five western policy contexts. Int J Child Care Educ Policy 2017;11:3. doi:10.1186/s40723-017-0028-8

17 Krapf S. Who uses public childcare for 2-year-old children? Coherent family policies and usage patterns in Sweden, Finland and Western Germany. Int J Soc Welf 2014;23:25-40. doi:10.1111/ijsw.12031

18 Heude B, Forhan A, Slama R, et al. Cohort Profile: The EDEN mother-child cohort on the prenatal and early postnatal determinants of child health and development. Int J Epidemiol 2016;45:35363. doi:10.1093/ije/dyv151

19 Shojaei T, Wazana A, Pitrou I, et al. The strengths and difficulties questionnaire: validation study in French school-aged children and cross-cultural comparisons. Soc Psychiatry Psychiatr Epidemiol 2008;44:740-7. doi:10.1007/s00127-008-0489-8

20 Youthinmind. Information for researchers and professionals about the Strengths \& Difficulties Questionnaires. 2015.http://www.youthinmind.com/ (accessed 8 Jun 2016).

21 Bernard JY, De Agostini M, Forhan A, et al. Breastfeeding duration and cognitive development at 2 and 3 years of age in the EDEN mother-child cohort. J Pediatr 2013;163:36-42.e1. doi:10.1016/j.jpeds.2012.11.090

22 Galera C, Heude B, Forhan A, et al. Prenatal diet and children's trajectories of hyperactivityinattention and conduct problems from 3 to 8 years: the EDEN mother-child cohort. J Child Psychol Psychiatry;0. doi:10.1111/jcpp.12898

23 Fuhrer R, Rouillon F. La version française de l'échelle CES-D (Center for Epidemiologic StudiesDepression Scale). Description et traduction de l'échelle d'autoévaluation. [The French version of the CES-D (Center for Epidemiologic Studies-Depression Scale).]. Eur Psychiatry 1989;4:163-6.

24 Matthey S, Henshaw C, Elliott S, et al. Variability in use of cut-off scores and formats on the Edinburgh Postnatal Depression Scale: implications for clinical and research practice. Arch Womens Ment Health 2006;9:309-15. doi:10.1007/s00737-006-0152-x

25 Nagin DS, Odgers CL. Group-Based Trajectory Modeling in Clinical Research. Annu Rev Clin Psychol 2010;6:109-38. doi:10.1146/annurev.clinpsy.121208.131413

26 Bartlett JW, Seaman SR, White IR, et al. Multiple imputation of covariates by fully conditional specification: Accommodating the substantive model. Stat Methods Med Res 2015;24:462-87. doi:10.1177/0962280214521348

27 Sterne JAC, White IR, Carlin JB, et al. Multiple imputation for missing data in epidemiological and clinical research: potential and pitfalls. BMJ 2009;338:b2393. doi:10.1136/bmj.b2393 
28 Lanehart RE, Rodriguez de Gil P, Kim ES, et al. Propensity Score Analysis and Assessment of Propensity Score Approaches Using SAS ${ }^{\circledR}$ Procedures.

2012.http://support.sas.com/resources/papers/proceedings12/314-2012.pdf (accessed $12 \mathrm{Jul}$ 2017).

29 Austin PC, Stuart EA. Moving towards best practice when using inverse probability of treatment weighting (IPTW) using the propensity score to estimate causal treatment effects in observational studies. Stat Med 2015;34:3661-79. doi:10.1002/sim.6607

30 Lavigne JV, Dahl KP, Gouze KR, et al. Multi-domain predictors of oppositional defiant disorder symptoms in preschool children: cross-informant differences. Child Psychiatry Hum Dev 2015;46:308-19. doi:10.1007/s10578-014-0472-4

31 Javo C, Rønning JA, Handegård BH, et al. Social competence and emotional/behavioral problems in a birth cohort of Sami and Norwegian preadolescents in Arctic Norway as reported by mothers and teachers. Nord J Psychiatry 2009;63:178-87. doi:10.1080/08039480902741752

32 OECD Country Note. Early Childhood Education and Care Policy in France. 2004.https://www.oecd.org/france/34400146.pdf (accessed 17 Jul 2017).

33 Tremblay RE, Nagin DS, Séguin JR, et al. Physical aggression during early childhood: trajectories and predictors. Can Child Adolesc Psychiatry Rev Rev Can Psychiatr Enfant Adolesc 2005;14:3-9.

34 Cepeda MS, Boston R, Farrar JT, et al. Comparison of logistic regression versus propensity score when the number of events is low and there are multiple confounders. Am J Epidemiol 2003;158:280-7.

35 Belsky J, Vandell DL, Burchinal M, et al. Are There Long-Term Effects of Early Child Care? Child Dev 2007;78:681-701. doi:10.1111/j.1467-8624.2007.01021.x

36 Zachrisson HD, Dearing E. Family income dynamics, early childhood education and care, and early child behavior problems in Norway. Child Dev 2015;86:425-40. doi:10.1111/cdev.12306

37 Collombet C. Qualité de l'accueil du jeune enfant en France et développement de l'enfant. 2012.https://www.caf.fr/sites/default/files/cnaf/Documents/international/divers/Note\%20quali t\%C3\%A9\%20de\%20l'accueil\%20ambassade\%20RU\%2030\%20nov\%20revue\%20DPFAS.pdf (accessed $12 \mathrm{Jul}$ 2017).

38 Bernal R, Fernández C. Subsidized childcare and child development in Colombia: Effects of Hogares Comunitarios de Bienestar as a function of timing and length of exposure. Soc Sci Med 2013;97:241-9. doi:10.1016/j.socscimed.2012.10.029

39 Klasen $\mathrm{H}$, Crombag A-C. What works where? A systematic review of child and adolescent mental health interventions for low and middle income countries. Soc Psychiatry Psychiatr Epidemiol 2013;48:595-611. doi:10.1007/s00127-012-0566-x

40 Walker SP, Wachs TD, Grantham-McGregor S, et al. Inequality in early childhood: risk and protective factors for early child development. The Lancet 2011;378:1325-38. doi:10.1016/S0140-6736(11)60555-2

41 Chaplin TM, Aldao A. Gender differences in emotion expression in children: a meta-analytic review. Psychol Bull 2013;139:735-65. doi:10.1037/a0030737 
42 Vandell DL, Burchinal M, Pierce KM. Early child care and adolescent functioning at the end of high school: Results from the NICHD Study of Early Child Care and Youth Development. Dev Psychol 2016;52:1634-45. doi:10.1037/dev0000169 


\section{Figures}

Figure 1: Children's emotional and behavioural symptoms from 3 to 8 years (EDEN cohort study, N = 1428, 2003-2011, France).
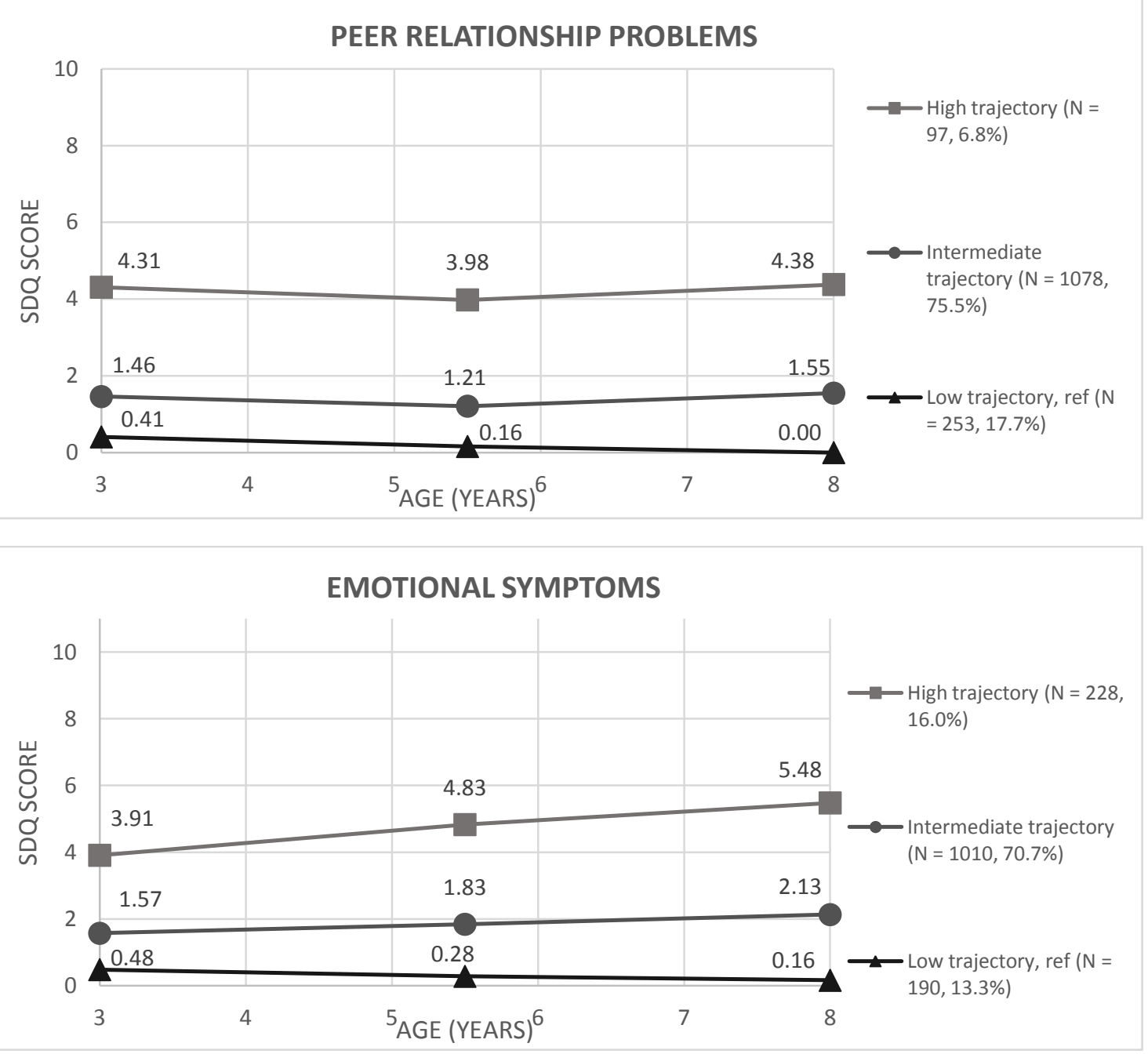
HYPERACTIVITY/INATTENTION
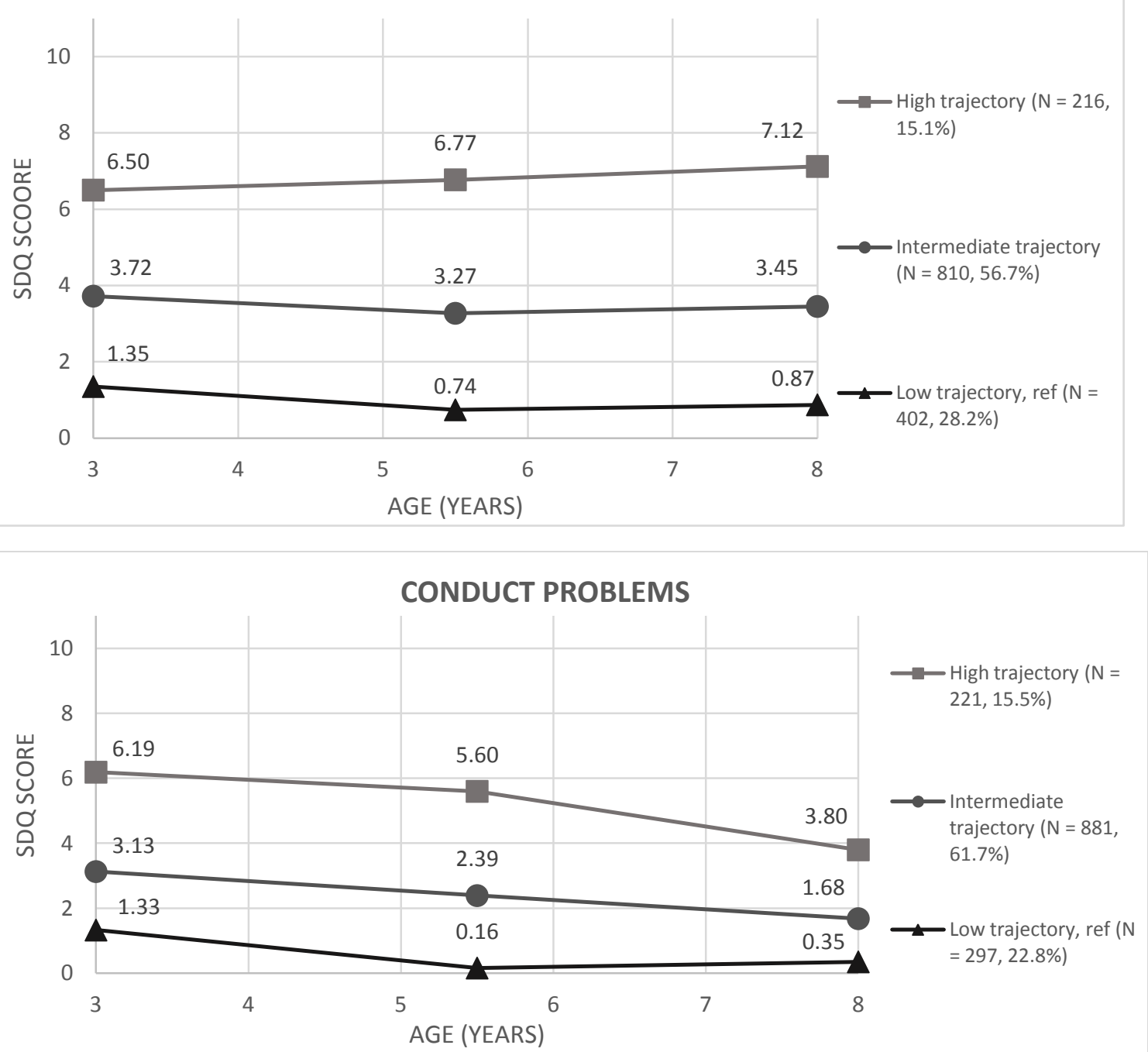

PROSOCIAL BEHAVIOUR

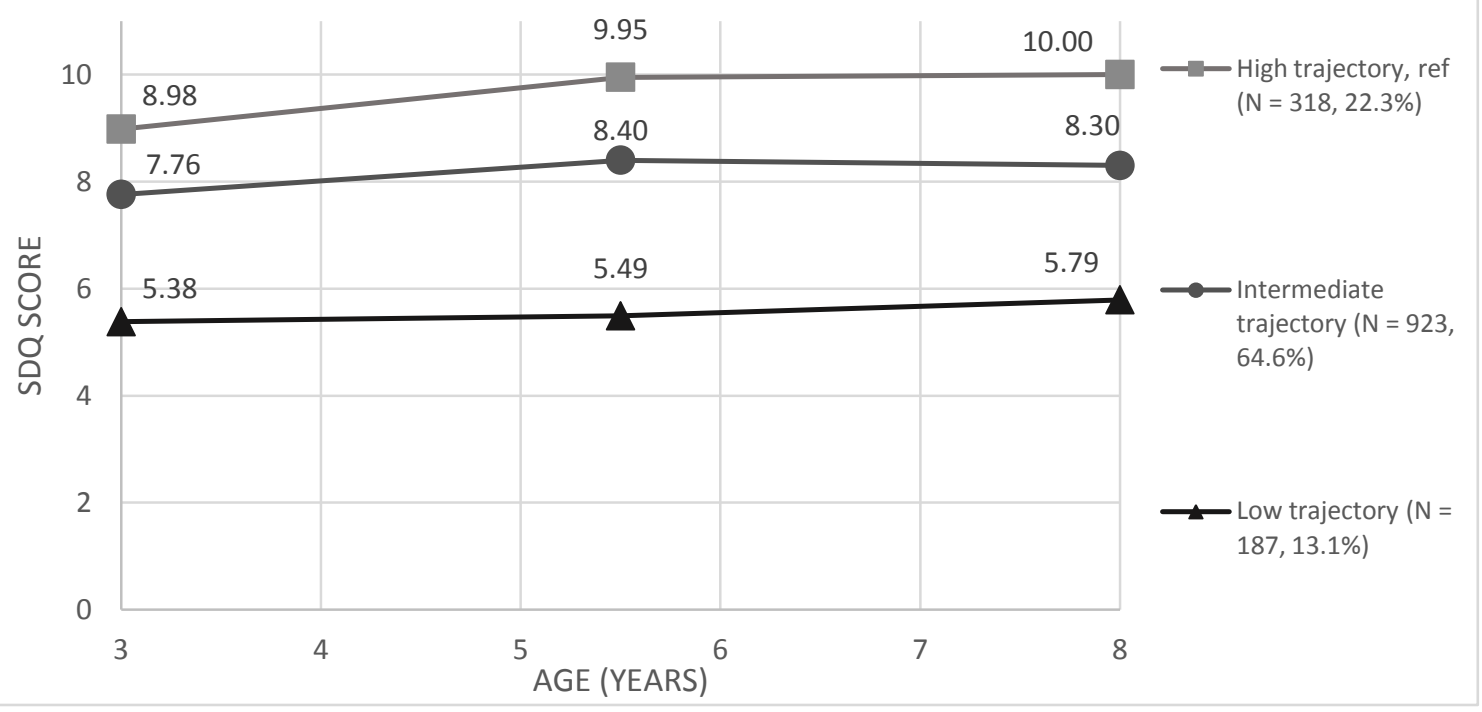


Figure 2: Early childcare (0-3 years) and children's behavioural and emotional difficulties (ages 3-8 years), stratifying by child sex, mother's education and depression. EDEN cohort study (n=1428), Inverse Probability Weight (IPW)-adjusted multinomial regression models $(95 \% \mathrm{CI})$
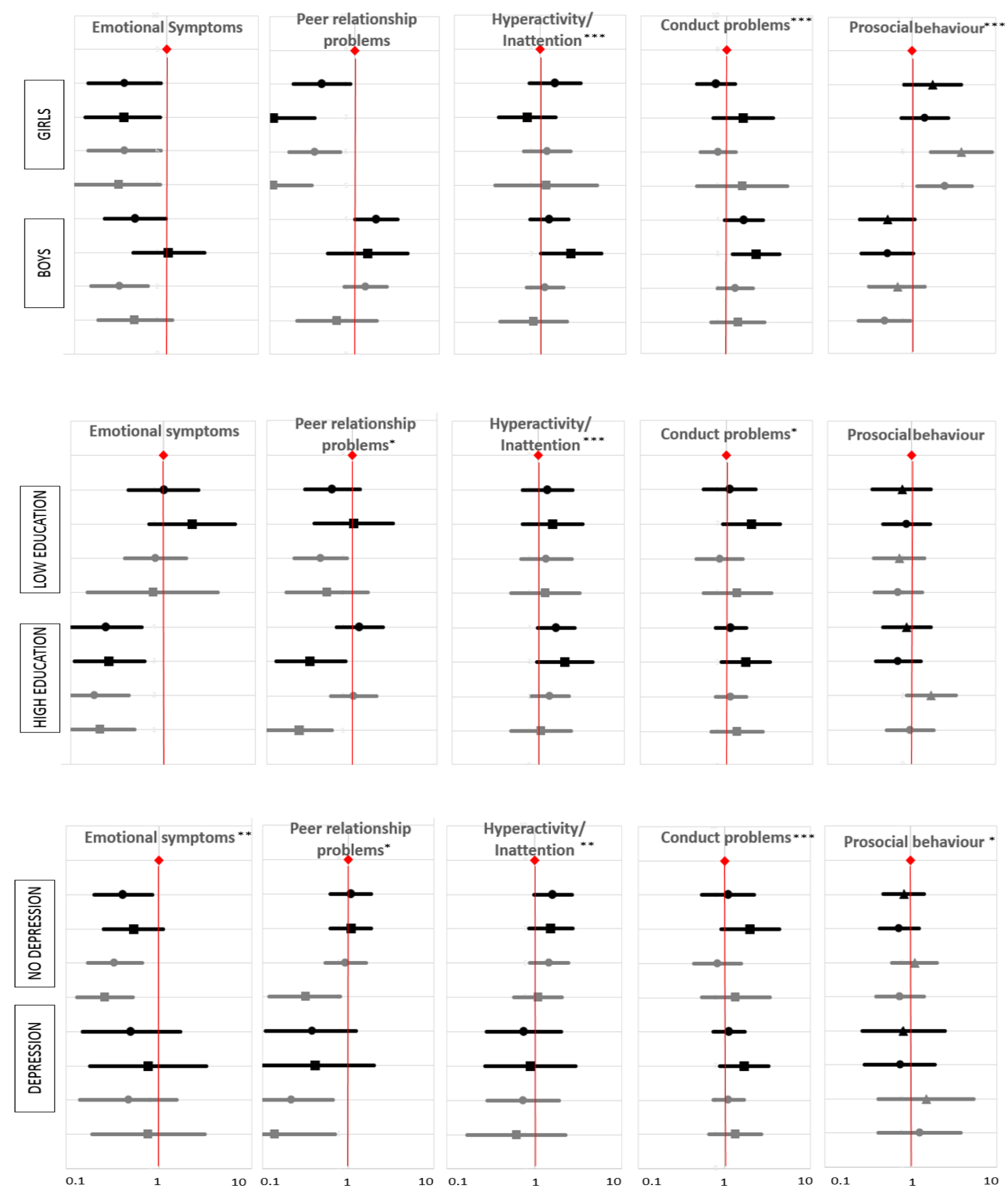

***: $\mathrm{p}$ value $<0.001 \quad * *$ : $\mathrm{p}$ between 0.001 and $0.01 \quad *$ : $\mathrm{p}$ value between 0.01 and 0.05 


\begin{tabular}{rlr|}
\hline Informal (reference) & $\leftarrow$ Childminder (Low) & - Centre-based (Low) \\
& $\rightarrow$ Childminder (Intermediate) & $\rightarrow$ Centre-based (Intermediate) \\
& $\rightarrow$ Childminder (High) & - -Centre-based (High) \\
\hline
\end{tabular}

